\title{
Configuration and Stability of HF/SF6 and HF/N2 Standard Gases
}

\author{
Zhang Shiling, ${ }^{1, *}$ \\ ${ }^{1}$ State Grid Chongqing Electric Power Company Chongqing Electric Power Research Institute, Chongqing, 401123, China
}

\begin{abstract}
In this paper, the analysis methods of HF components in two background series of nitrogen and sulfur hexafluoride were established and optimized. The uncertainty contribution in the weighing process was evaluated. The configuration of HF/SF6 and HF/N2 standard gases was established, that is, the packaging volume was $4 \mathrm{~L}$ and $8 \mathrm{~L}$, and the content range of HF component was $(50-100) \times 10^{-6} \mathrm{~mol} / \mathrm{mol}$. Then, the stability of the value of the standard gas is investigated, that is, the stability of the value with time and pressure, the fixed value and performance investigation. The relative expanded uncertainty of two series HF standard gas prediction is less than $6 \%(\mathrm{k}=2)$.
\end{abstract}

\section{Introduction}

For the purity of HF pure gas, the supplier takes into account the influence of inorganic elements and metal ions on its purity according to the production process. In anhydrous hydrogen fluoride, we consider the influence of impurity content in gaseous form on the purity of HF. We need to establish relevant analysis methods for the measurement of the gaseous impurity components. In the initial stage of development, considering the physical and the chemical characteristics of the hydrogen fluoride components, the gas cylinders was selected before preparation, that is, analyzing and selecting cylinders with relatively stable inner wall materials for hydrogen fluoride. Based on the analysis and investigation of several existing gas cylinders, packaging gas cylinders are optimized[1,2].

According to the special chemical properties of $\mathrm{HF}$, it is necessary to investigate the inert strength of inner wall material of gas cylinder to HF before the preparation of standard gas. After selecting PC type gas cylinder, first vacuum the cylinder at $80^{\circ} \mathrm{C}$ for 2 hours, then vacuum it at room temperature for 12 hours, and then fill the cylinder with about 2000ppm HF/N2, adjust the pressure to 10bar, and then place it for saturation. After 24-48 hours and at different time after that, Fourier transform infrared spectrometer was used to detect bottle by bottle and record the response value[3]. During the whole investigation period, the test conditions and parameter requirements are consistent. According to the change of response value in different time, the inert strength of inner wall material to HF was evaluated. The cylinder which meets the requirement of inert strength can be used as the packaging container of HF standard gas.

In the process of stability investigation of characteristic value of standard gas, it is necessary to establish the appropriate detection method. According to the literature and the detection experience, ion chromatography and Fourier transform infrared spectroscopy are commonly used to measure $\mathrm{HF}$ components. The working principle of Fourier transform infrared spectrometer is: the light emitted by the light source is divided into two beams by the beam splitter (similar to semi transparent and semi reflective mirror). One beam reaches the moving mirror through transmission, and the other reaches the fixed mirror through reflection. Two beams of light are reflected by the fixed mirror and the moving mirror respectively, and then return to the beam splitter. The moving mirror moves in the straight line at constant speed[4,5]. Therefore, the two beams of light separated by the beam splitter form optical path difference and interfere. Interference light passes through the sample cell after the beam splitter converges. After passing through the sample, the interference light containing the sample information reaches the detector. Then the signal is processed by the Fourier transform, and finally the infrared absorption spectrum of the transmittance or absorbance with wave number or wavelength is obtained.

\section{Precision test of analytical method}

The response values of the HF gas reference materials with different concentrations were measured, and the samples of each concentration point were injected 10 times continuously. Record and average the analytical response value of the instrument, and calculate the relative standard deviation (RSD\%) of the average value[6]. Based on this, it can be judged whether the precision of the analytical method can meet the needs of the stability investigation of the characteristic value of the standard gas.

It can be seen from Table 1 that the repeatability relative standard deviation (RSD) of HF gas reference materials established by the project research institute is less than $0.8 \%$, which can meet the analysis requirements of this project. 
Table 1. Analysis accuracy measurement results of HF / N2 by gas chromatography.

\begin{tabular}{|c|c|c|c|c|}
\hline \multirow{2}{*}{$\begin{array}{c}1 \text { XP EH } \\
\square\end{array}$} & \multicolumn{4}{|c|}{ Cylinder number } \\
\hline & L1612021 & L172403 & L1950081 & L195008181 \\
\hline & $30(4 \mathrm{~L})$ & $153(4 \mathrm{~L})$ & $76(8 \mathrm{~L})$ & $(8 \mathrm{~L})$ \\
\hline & $31.40 \mathrm{ppm}$ & $57.10 \mathrm{ppm}$ & $53.90 \mathrm{ppm}$ & $55.65 \mathrm{ppm}$ \\
\hline \multicolumn{5}{|l|}{ Content } \\
\hline 1 & 14.695 & 26.639 & 24.530 & 25.188 \\
\hline 2 & 14.734 & 26.646 & 24.726 & 25.324 \\
\hline 3 & 14.756 & 26.611 & 24.961 & 25.401 \\
\hline 4 & 14.812 & 26.678 & 24.942 & 25.445 \\
\hline 5 & 14.722 & 26.951 & 24.904 & 25.528 \\
\hline 6 & 14.870 & 26.733 & 24.818 & 25.491 \\
\hline 7 & 14.827 & 26.607 & 25.180 & 25.390 \\
\hline 8 & 14.939 & 26.819 & 25.037 & 25.361 \\
\hline 9 & 14.927 & 26.849 & 24.621 & 25.538 \\
\hline 10 & 14.911 & 26.882 & 24.608 & 25.709 \\
\hline $\begin{array}{c}\text { Standard } \\
\text { deviation(S } \\
)\end{array}$ & 0.090 & 0.125 & 0.184 & 0.141 \\
\hline $\begin{array}{l}\text { Average } \\
\text { value }\end{array}$ & 14.819 & 26.742 & 24.817 & 25.438 \\
\hline $\operatorname{RSD}(\%)$ & 0.61 & 0.47 & 0.74 & 0.56 \\
\hline NumbH & \multicolumn{4}{|c|}{ Cylinder number } \\
\hline & L5351704 & L183101 & L1871010 & L187208112 \\
\hline & 7 & $090(8 \mathrm{~L})$ & $42(8 \mathrm{~L})$ & $(8 \mathrm{~L})$ \\
\hline Content & $(4 \mathrm{~L})$ & & & \\
\hline & $101.1 \mathrm{ppm}$ & $107.3 \mathrm{ppm}$ & 99.90ppm & $100.5 \mathrm{ppm}$ \\
\hline 1 & 42.173 & 46.677 & 38.929 & 42.545 \\
\hline 2 & 42.236 & 46.420 & 38.792 & 42.442 \\
\hline 3 & 42.358 & 46.838 & 38.774 & 42.354 \\
\hline 4 & 42.311 & 46.593 & 39.139 & 42.168 \\
\hline 5 & 42,234 & 46.822 & 39.246 & 42.374 \\
\hline 6 & 42.305 & 46.972 & 39.34 & 42.609 \\
\hline 7 & 42.196 & 46.734 & 39.452 & 42.656 \\
\hline 8 & 42.281 & 46.926 & 39.484 & 42.507 \\
\hline 9 & 42.315 & 46.555 & 39.563 & 42.171 \\
\hline 10 & 42.433 & 46.657 & 39.469 & 42.694 \\
\hline $\begin{array}{c}\text { Standard } \\
\text { deviation(S } \\
)\end{array}$ & 0.078 & 0.173 & 0.297 & 0.186 \\
\hline $\begin{array}{c}\text { Average } \\
\text { value }\end{array}$ & 42.284 & 46.719 & 39.219 & 42.452 \\
\hline RSD(\%) & 0.19 & 0.37 & 0.77 & 0.44 \\
\hline
\end{tabular}

\section{Long term stability investigation}

In order to investigate the long-term stability of the component values of the developed gas reference materials under room temperature storage conditions, the component values of the prepared gas mixture were tracked with time[7]. The test design: according to the international standard ISO guide 35, long-term stability of the prepared gas reference material is tested by the classical stability research method (trend analysis). Three bottles of each concentration point were selected for the long-term stability test. The test results will be statistically processed to check whether the value has the significant change trend with time, and the uncertainty of stability will be evaluated. The evaluation result depends on all the data of the whole investigation period, and there is no result at present.

In the project study, the stability of different pressure points is designed for the study of the variation of characteristic value of standard gas with pressure. In other words, the test points are selected within the range of the filling upper limit pressure to possible minimum working pressure, four pressure points are set for $\mathrm{HF} / \mathrm{N} 2$, the pressure points of standard gas are 90bar, 70bar, 50bar and 20bar. For HF/SF6, four pressure points are set, the pressure points of standard gas are 12bar, 10bar, 5 bar and 2 bar.

For the stability investigation of the characteristic value of standard gas, it needs to be supplemented because the inspection time does not meet the design requirements. According to the GGF1344-2012 general technical requirements for the development (production) of the gas reference materials, three sources of the uncertainty are considered in "determination of characteristic value of synthetic RM and mixed gas".

The uncertainty introduced by the gas distribution by the weighing method is expressed as R. The uncertainty introduced by pressure stability is expressed as UPS, R. if the pressure release test result is not significant through $\mathrm{F}$ test, the uncertainty introduced by pressure stability can be ignored. The uncertainty introduced by the long-term stability is shown as ults, R. Therefore, the uncertainty of final fixed value can be obtained by the following formula:

$$
u_{r e f, r}=\sqrt{u_{g r a v, r}^{2}+u_{l t s, r}^{2}}
$$

Relative expanded uncertainty: Uref, $\mathrm{r}=$ kuref, $\mathrm{r}(\mathrm{k}=2$, Confidence probability $=95 \%$ )

\section{Test result}

The development team determined following high purity anhydrous hydrogen fluoride, as shown in Figure 1. After gas purity analysis and optimization of packaging container selection, according to the ISO 6142 . HF/N2 standard gases were prepared according to the gas distribution process. The standard gases prepared by weighing method are shown in Table 2.

The HF gas reference material is introduced into the instrument through the sample acquisition system, and the stable response information is obtained under the conditions of the stable sample flow rate and stable gas pressure in the gas pool. 


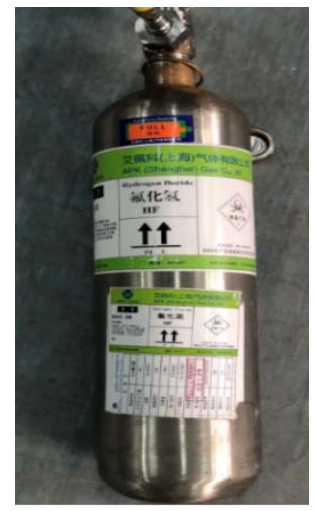

Figure 1. High purity anhydrous hydrogen fluoride. Table 2. List of HF gas reference materials.

\begin{tabular}{|c|c|c|c|c|}
\hline $\begin{array}{c}\text { Name of } \\
\text { standard } \\
\text { gas }\end{array}$ & $\begin{array}{l}\text { Bottle } \\
\text { number }\end{array}$ & $\begin{array}{c}\text { Component } \\
\text { value } \\
(\mu \mathrm{mol} / \mathrm{mol})\end{array}$ & $\begin{array}{l}\text { volume } \\
\text { (L) }\end{array}$ & $\begin{array}{c}\text { Filling } \\
\text { Pressure } \\
\text { (bar) }\end{array}$ \\
\hline \multirow{8}{*}{ HF6/N2 } & L53516017 & 49.99 & 8 & 90 \\
\hline & L53516002 & 50.01 & 8 & 90 \\
\hline & 94909112 & 49.96 & 8 & 90 \\
\hline & 94907065 & 50.00 & 8 & 90 \\
\hline & 92113141 & 100.1 & 4 & 90 \\
\hline & 85412187 & 100.0 & 4 & 90 \\
\hline & 92113193 & 107.1 & 4 & 90 \\
\hline & 92113036 & 93.10 & 4 & 90 \\
\hline \multirow{8}{*}{ HF6/SF6 } & \multirow{8}{*}{$\begin{array}{c}\text { In } \\
\text { preparation }\end{array}$} & $\sim 50$ & 8 & 12 \\
\hline & & $\sim 50$ & 8 & 12 \\
\hline & & $\sim 50$ & 8 & 12 \\
\hline & & $\sim 50$ & 8 & 12 \\
\hline & & $\sim 100$ & 8 & 12 \\
\hline & & $\sim 100$ & 8 & 12 \\
\hline & & $\sim 100$ & 8 & 12 \\
\hline & & $\sim 100$ & 8 & 12 \\
\hline
\end{tabular}

The concentration of each sample was measured for 10 times. Record and average the analytical response value of the instrument, and calculate the relative standard deviation (RSD\%) of the average value[8,9]. Based on this, it can be judged whether precision of the analytical method can meet the needs of the stability investigation of the characteristic value of the standard gas.
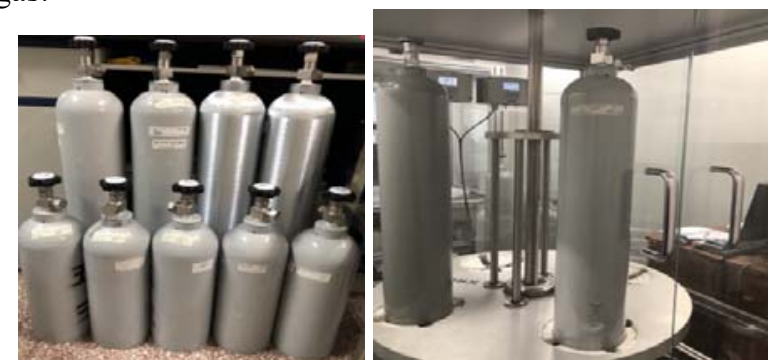

Figure 2. Automatic weighing device by substitution method.

It can be seen from the experimental results that the repeatability relative standard deviation (RSD) of HF gas reference materials established by the project research institute is less than $0.8 \%$, which can meet the analysis requirements of this project.

\section{Field application of HF standard gas}

In the presence of water and other impurities, once high voltage discharge occurs, SF6 may have the irreversible reaction and produce decomposition products such as $\mathrm{SO} 2, \mathrm{H} 2 \mathrm{~S}, \mathrm{HF}[10]$. In general, excessive adsorbent will be set in SF6 high-pressure equipment to absorb these impurities. HF gas, the research object of this project, is easy to be adsorbed by adsorbent because of its active chemical properties and strong physical adsorption. If the HF gas detection optical path is set on the flange surface, the final measurement results will be affected by the influence of adsorbent adsorption during the process of HF gas diffusion to the optical path surface after the generation of HF gas. The measured data is very easy to be greatly low, which can not accurately characterize the amount of HF gas generated in the discharge process.

Considering that the measurement process of the optical path cell with flange plate needs gas diffusion, it may be delayed. In order to realize on-line measurement of high-voltage equipment, the project team invented an on-line detection optical structure. The whole optical route is composed of the laser detection optical path, reflection optical path, signal processing and display system. They are fixed together with the high-voltage switch-gear on site through the flange to realize real-time monitoring of field data. The structure diagram is shown in Figure 3. The design of the whole structure light path is mainly to improve the reliability and practicability of the system. The beam of light emitted by the laser is projected onto the window plate after passing collimator. The window plate mainly plays the role of isolating the measured gas and air. After refraction, the laser enters the high-voltage electrical equipment, passes through the target gas, and then passes through the corner cube prism to make the light reflect back in parallel. The reflected light passes through the window again and reaches the off-axis mirror. After being reflected and focused by offaxis paraboloid mirror, the light is reflected to the infrared detector, and the detector converts the received optical signal into the electrical signal for the subsequent signal processing and data conversion.

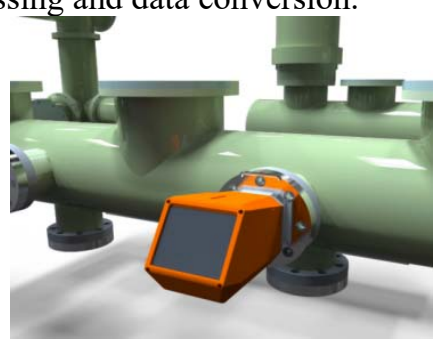

Figure 3. Rendering effect of online HF detection device.

In order to ensure that there is no safety accident during the project experiment, project team decided to install the equipment in the laboratory high-voltage switch body after analysis and research, and then carry out the field verification after the stage experiment. 


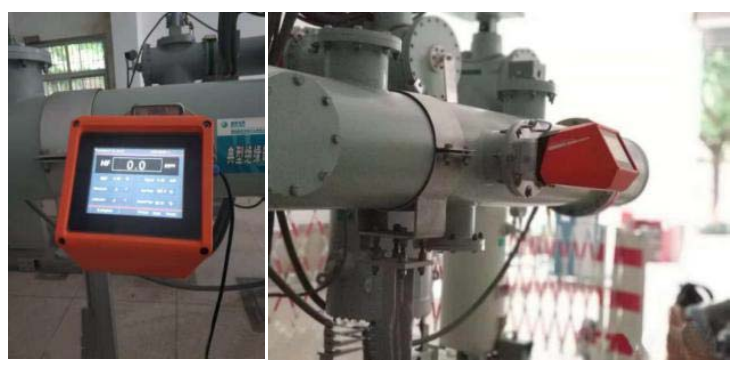

Figure 4. Equipment installation location selection.

After installation which is shown in Figure 4, adjust the optical signal. Firstly, the infrared laser is connected for the calibration, and the adjustment device is adjusted to make the red light focus on the infrared detector. Then connect the HF detection infrared laser, observe the laser receiving board, make the infrared light spot focus to the center of the receiving board as far as possible, connect the debugging board and the multimeter, make the photoelectric conversion signal greater than $2 \mathrm{~mA}$, observe the gas absorption peak signal-to-noise ratio is good, judge that the equipment is working normally, so far the equipment installation and debugging are completed.

\section{Conclusion}

1)In the project study, the stability of different pressure points is designed for the study of the variation of characteristic value of standard gas with pressure. In other words, the test points are selected within the range of the filling upper limit pressure to possible minimum working pressure, four pressure points are set for HF/ N2, the pressure points of standard gas are 90bar, 70bar, 50bar and 20bar. For HF/SF6, four pressure points are set, the pressure points of standard gas are 12bar, 10bar, 5 bar and 2 bar.

2)In order to investigate the long-term stability of the component values of developed gas reference materials under the room temperature storage conditions, the component values of the prepared gas mixture were tracked with time. The test design: according to the international standard ISO guide 35, the long-term stability of the prepared gas reference material is tested by the classical stability research method (trend analysis). Three bottles of each concentration point were selected for long-term stability test. The test results will be statistically processed to check whether the value has a significant change trend with time, and the uncertainty of stability will be evaluated.

3)In order to realize on-line measurement, the project team developed a detection structure based on the optical path. After the development of the project team, a series of experiments were carried out on the simulation device, and the problems such as on-line detection and the reduction of harmonic amplitude caused by pressure broadening were deeply studied. Finally, the equipment was installed and tested in high-voltage equipment.

\section{References}

1. BrassingtonDJ, Spectroscopy in Environmental
Science, eds. HseterRE, ClarkR J, 1995， 43-87.

2. P.Werle, A review of recent advances in semiconductor laser based gas monitors, Spectro chimica Acta, PartA, 1998, 54:197-236.

3. SAVADKOUHIJ.Reliability considerations for gas delivery components[C] //Advanced Semiconductor Manufacturing Conference and Workshop.IEEE/SEM, USA, 2003: 255-261.

4. R.R.Gamache, S.Kennedy, R.Hawkinsetal. Total internal partition sums form Olecules in the terrestrial atmosphere. Journal of Molecular Structure. 2000, 517(1):407-425.

5. J.Fischer, R.R.Gamache, A.Goldman, etal.Total internal partition sums for mol Ecularspeciesinthe2000editionoftheHITRANdataba se.Journal of Quantitative Spectroscopyand Radiative Transfer, 2003, 82(1), 401-412.

6. LAUTIERA, DEHET.GAILLARDD. Control of gaseous mixture in membrane oxygenators , Engineering in Medicineand Biology Society. Proceedings of the Annual International Conference of the IEEE, France, 1998:838-839.

7. EmilZak, Jonathan.T, Polyansky.O, et.al. A room temperature $\mathrm{CO} 2$ line list with abinitio computed intensities $[\mathrm{J}]$. Journal of Quantitative Spectroscopy and Radiative, Transfer, 2016, 177(7):31-42.

8. Jagadeeshwari, M.Alan.L , John.T , et.al. Wavelength modulation spectroscopy with a pulsed quantum cascade laser for the sensitive detection of acrylonitrile[J] . APPLIED OPTICS , 2011 , 50(25): 112-118.

9. Ma.Y.F, Lewicki.R, Razeghi.M, et.al. QEPAS based ppb-level detection of $\mathrm{CO}$, and $\mathrm{N} 2 \mathrm{O}$ using a high power CW DFB-QCL. Optics Express, 2013, 21(1): 1008-1019.

10. HE.Ying, ZHANG.Yu-jun, YOU.Kun, et .al. Study on Hydrogen Fluoride at High Temperature Detection Method with Temperature Correction Based on Laser Technology[J] .Spectroscopy and Spectral Analysis, 2017, 37(3): 964-970. 